\title{
Editorials
}

\section{Tracheal intubation: optimal conditions, vocal cord damage, and allergy}

François Donati PhD MD FrCPC, * Benoît Plaud MD PhD†

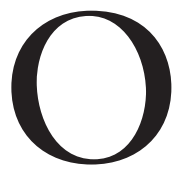
ne of the indications of neuromuscular blocking drugs (NMBDs) is to provide adequate conditions for performing tracheal intubation. However, many studies have shown that tracheal intubation can be successfully performed without NMBDs, by using an appropriate mixture of an intravenous or inhaled hypnotic, an opioid, and possibly another adjuvant, such as lidocaine. It is impossible to determine the regimen that will provide the optimal conditions, with minimal side effects, as the combinations of agents, doses, and timing of administration are almost endless. To compound the problem, in spite of attempts at standardization, assessment of intubating conditions is somewhat subjective. ${ }^{1,2}$

Proponents of intubation without paralyzing agents mention that NMBDs are not always indicated for the surgical procedure. Their duration of action may be too long for the intended surgery, or there may be residual paralysis, as stated by Bouvet et al. ${ }^{3}$ in this issue of the Journal. Also, these authors mention the possibility of allergic or anaphylactic reactions as the principal reason for avoiding NMBDs. Of note, the Bouvet et al. study was conducted in France, where anaphylaxis during anesthesia is a great concern. Those who favour the almost systematic use of NMBDs for tracheal intubation argue that aiming for high quality intubating conditions is not only a matter of elegance, but also a question of patient safety. Poor intubating conditions may be associated with cough, movement, ventilation difficulties, oxygen desaturation, and an increased risk of pulmonary aspiration. ${ }^{4}$ Recently, poor conditions have been associated with complaints of hoarseness and sore throat in the postoperative period, with documentation of laryngeal injuries. ${ }^{5}$ Given the evidence presented by Bouvet et $a l^{3}$ and considering previous studies, should we be more concerned about paralyzing our patients or damaging their vocal cords?

\section{Intubating conditions}

Until the mid-1990's, investigators comparing the ability of two induction regimens on intubating conditions had to either develop their own scoring system or adopt a previously proposed classification. In 1994, a group of experts met in Denmark to propose a standardization of many aspects of the studies on NMBDs, including intubating conditions. The recommendations of this group were published in 1996, ${ }^{1}$ and the intubating scoring system the experts proposed has since been adopted by virtually all investigators, including Bouvet et al. ${ }^{3}$ The initial recommendations were updated at a meeting held in Stockholm in 2005 and were published last year. ${ }^{2}$ Unfortunately, the updated recommendations relating to intubating conditions lack the clarity and detail of the original edition; therefore, it appears preferable to adhere to the previous scoring system for future studies.

The original system, introduced in 1996 to grade intubating conditions, is based on an assessment, completed by a blinded investigator, of the following five items: ease of laryngoscopy, vocal cord position, vocal cord movement, limb movement, and cough. Each item is graded as excellent, good, or poor, according to specific criteria. For example, the item "limb movement" is rated excellent, if there is no movement at all;

CAN J ANESTH 2008 / 55: $10 /$ pp 663-669

From the Département d'anesthésiologie, * Hôpital Maisonneuve-Rosemont and Université de Montréal, Montréal, Québec, Canada; and the Pôle anesthésie, réanimation chirurgicale, $†$ Université de Caen Basse Normandie and CHU de la Côte de Nacre, Caen, France. Address correspondence to: Dr. François Donati, Département d'anesthésiologie, Hôpital Maisonneuve-Rosemont, 5415, boul

l'Assomption, Montréal, Québec HIT 2M4, Canada. Phone: 514-252-3426; Fax: 514-252-3542; E-mail: francois.donati@umontreal.ca Competing interests: None declared. 
good, if movement is "slight"; and poor, if movement is "vigorous". As it is relatively easy to assess if there is movement or not, an excellent score is easily separated from the other grades. However, the line between "slight" and "vigorous" movement might be markedly observer-dependent, making it harder to separate "good" from "poor". The final score depends on the worst score of any item. Consequently, the final score is considered poor, if any item is rated poor; it is good, if one or more items are rated good and none are rated poor; and it is excellent, only if all items are judged excellent.

In many studies, such as the study completed by Bouvet et al., ${ }^{3}$ excellent and good scores are pooled into an "acceptable" category, while a poor score qualifies as "unacceptable". Thus, the boundary between acceptable and unacceptable conditions is somewhat blurred and may be assessor-dependent. While there could be consistency within a study, comparisons between studies or extension to clinical practice may be difficult. For example, Bouvet et al. ${ }^{3}$ found "acceptable" intubating conditions to be present in $95 \%$ of subjects without NMBDs. It is possible that some "good" scores $(45 \%$ of patients) may have been rated as "poor" by another assessor, because of blurring between the "good" and "poor" categories. In the cisatracurium group, however, only $17 \%$ of subjects received a "good" score; the remainder were rated "excellent". As a result, there are fewer instances where another observer would have rated the conditions as "poor", as it is unlikely that one observer would give an "excellent" rating, while another observer would give a "poor" rating to the same event. Excellent conditions are less subject to inter-observer variability, since they only occur when there is no movement at all, and there is usually consistency in the incidence of excellent conditions across studies. For example, at one minute after succinylcholine administration, $\mathrm{l} \mathrm{mg} \cdot \mathrm{kg}^{-1}{ }^{6}$ most studies find excellent intubating conditions in $70-80 \%$ of patients.

Quite apart from the fact that an "excellent" rating is better for our patients than merely a "good" rating, discriminating between excellent and good conditions allows the design of studies with more refined outcome measures, using smaller numbers of patients. For example, in the study by Bouvet et al. ${ }^{3}$ the rates of acceptable conditions were not statistically different between the groups, $100 \%$ and $95 \%$ in the paralyzed and non-paralyzed groups, respectively. If, indeed, the real difference is $5 \%$, one would require over 800 patients per group to detect a statistically significant difference. If all three categories are considered, however, a re-analysis of the data suggests that intubating conditions are better in the group that received cisatracurium $(P<0.001)$. Is this information, obtained with 65 patients per group, clinically relevant? Yes, in all likelihood, it is. If excellent conditions occur more often, chances are that poor conditions will occur less frequently. Furthermore, if the rate of poor conditions is only $5 \%$, this event will be observed 50 times by someone who performs 1,000 tracheal intubations in a year!

\section{Vocal cord damage}

Tracheal intubation is very rarely associated with severe laryngeal or tracheal symptoms, but these events do occur and are associated with difficult intubation. ${ }^{7}$ The number of patients needed to determine whether intubation without NMBDs leads to more or fewer of these injuries would be very large indeed. However, tracheal intubation leads to minor, yet frequent, symptoms, such as hoarseness and sore throat, and it is likely that a procedure that yields more of these minor side effects will also be associated with more severe, yet infrequent, outcomes, just as a high incidence of only "good" intubating conditions indicates an increased likelihood of "poor" conditions. This approach was first undertaken by Mencke et al. ${ }^{5}$ who compared postoperative sore throat and hoarseness in patients who had their tracheas intubated both with and without NMBDs. Vocal cord damage was assessed by an experienced otolaryngologist using a fibreoptic examination. Not surprisingly, intubating conditions were superior with an NMBD (in that case atracurium) than with saline, with $43 \%$ and $5 \%$ excellent intubating conditions, respectively. The incidence of hoarseness was greater in the saline group, and correlated well with the poor quality of intubating conditions. Poor intubating conditions were associated with a $40 \%$ incidence of vocal cord sequelae, compared with fewer than $20 \%$ in the "excellent" group. The authors of that study concluded that, "the quality of tracheal intubation may affect the incidence of laryngeal morbidity." 5

Two additional studies were performed on laryngeal injuries by the same research group, but all patients received neuromuscular blocking agents. ${ }^{8,9}$ No statistically significant difference in hoarseness, sore throat, or cord injury was found between groups, probably because the incidence of poor intubating conditions was not very different between groups, and other factors, such as coughing at extubation, may also have contributed to postoperative laryngeal side effects. In the study by Bouvet et al., the incidence of vocal cord sequelae was very low in both groups, but only patients with persisting symptoms were examined. The incidence of hoarseness at $24 \mathrm{hr}$ was $20-30 \%$ in both groups, which corresponds to the incidence reported 
by Mencke et al..$^{5}$ in their first study in patients with excellent or good intubating conditions.

\section{Anaphylaxis}

Considering that the use of NMBDs improves intubating conditions and may possibly decrease the incidence of laryngeal symptoms, why is it that some anesthesiologists advocate omitting paralyzing agents and express an interest in methods that do not involve these drugs? For short duration procedures, using a small dose of an NMBD yields better intubating conditions than using nothing at all. ${ }^{10}$ Postoperative residual paralysis can be effectively reduced by reversal agents and appropriate monitoring. ${ }^{11}$ Bouvet et al. ${ }^{3}$ mention the risk of anaphylactic reactions, which is perceived as a major concern in certain countries, particularly in France and Norway.

The French obsession with anaphylactic reactions may appear puzzling to North American and other European observers, while the lack of concern in North America for possible allergic reactions to NMBDs might seem unreal to French anesthesiologists. In France, a very extensive reporting network has been developed over the past 20 years. ${ }^{12,13}$ It is difficult to determine the incidence of anaphylactic reactions from such surveys, because reporting may be incomplete, the denominator is uncertain, and the reliability of the tests is not known. Still, the overall incidence has been estimated to be anywhere between 1:3,000 and 1:20,000 anesthetics, with more frequent occurrences, possibly as high as $1: 1,500$, for allegedly "allergenic" drugs such as succinylcholine and rocuronium. ${ }^{12-14}$

Interest in allergic reactions has also developed in Australia, the United Kingdom, and Scandinavian countries. In Norway, rocuronium was virtually eliminated from the anesthesia carts after an estimated 150,000 administrations led to 29 reports of anaphylactic reactions in $1997-2000(1: 5,172) .{ }^{15}$ Succinylcholine was also frequently implicated in allergic reactions. During the same period, only seven such reports appeared in neighbouring Sweden, Finland, and Denmark, following an estimated 800,000 rocuronium doses $(1: 114,000)$. An allergy referral centre in Denmark reported that, contrary to the situation in France, the most common agent implicated in allergic reactions was chlorhexidine, not NMBDs. ${ }^{16}$ In the United States, reports of allergic reactions to the Food and Drug Administration are very rare (approximately $1: 1,000,000$ administrations of rocuronium). ${ }^{17}$

While some would attribute these widely different incidence rates to underreporting in some countries, or perhaps to statistical variations, ${ }^{16}$ others hypothesized that environmental factors could play a role.
A study was undertaken to screen products available in Norway, but not in the other Scandinavian countries, that could sensitize patients to NMBDs. ${ }^{18}$ Succinylcholine IgE antibodies were found in $0.4 \%$ of Norwegian blood donors. but in none of the Swedish donors. After screening many products, the authors of that study focused on pholcodine, an antitussive found in over-the-counter cough syrups in Norway, but unavailable in Sweden. Sensitization to pholcodine was common in Norway, but virtually absent in Sweden, and cross-sensitization with neuromuscular blocking agents could occur. Interestingly, pholcodine is available in France, the United Kingdom, and Australia, where there has been considerable interest in allergic reactions, but it is not available in Canada or in the United States. ${ }^{18}$

Does pholcodine explain the apparent differences in the incidence anaphylactic reactions between countries? At present, the evidence is circumstantial, but the lead is interesting. Other factors could be involved, and over-reporting could play a role. In any event, it is interesting to consider that something as unlikely as the presence or absence of an over-the-counter cough syrup in the community might influence the manner in which we approach tracheal intubation and the practice of anesthesia.

\section{Conclusion}

Hoarseness and laryngeal trauma are frequent and have been linked to poor tracheal intubating conditions. In all likelihood, these minor side effects are indicators of less frequent, but more severe, complications of tracheal intubation. Similarly, an induction sequence that often yields good rather than excellent intubating conditions is more likely to provide poor intubating conditions in some patients than a regimen that yields excellent intubating conditions in most subjects. Thus, aiming for excellent, rather than good, intubating conditions reduces the probability of complications such as laryngeal injuries, and the simplest way to achieve this result is to use NMBDs. The most compelling reason to omit paralyzing agents is the perceived concern for allergic reactions, and there is some weak evidence that the propensity to develop such reactions might be country-specific. Unfortunately, a milder, more frequent form of allergic reaction has not been described that could be used as a marker for more severe events, as is the case of laryngeal morbidity. Anesthesiologists who use NMBDs to facilitate tracheal intubation should continue to do so: if the incidence of allergic reactions were $>1: 5,000$ in a given region, there would be, at the very least, an annual occurrence of such events in a moderately 
sized hospital, and individual practitioners would be aware of the problem. Those practitioners who choose to withhold NMBDs at the time of tracheal intubation, because of possible anaphylactic reactions, must develop the skill and experience to aim for excellent, rather than good, intubating conditions.

\section{Intubation trachéale: conditions optimales, lésions laryngées et allergie}

Une des indications des curares est de fournir des conditions appropriées pour effectuer une intubation trachéale. Toutefois, de nombreuses études ont établi que l'intubation trachéale pouvait être réalisée sans curare, en utilisant un mélange approprié d'un hypnotique intraveineux ou inhalé, d'un opiacé et au besoin d'un autre adjuvant, comme la lidocaïne. Il est impossible de déterminer la recette qui donnera les conditions idéales, parce que les combinaisons de médicaments, de doses et de séquence temporelle d'administration sont en nombre presque infini. Pour compliquer les choses, l'évaluation des conditions d'intubation est de nature subjective, malgré des efforts de standardisation. ${ }^{1,2}$

Ceux qui préconisent l'intubation sans curarisation avancent que les curares ne sont pas toujours indiqués pour l'intervention chirurgicale, que leur durée d'action pourrait perdurer au-delà de la fin du geste chirurgical ou qu'il existe un risque de curarisation résiduelle, comme le précisent Bouvet et coll. ${ }^{3}$ dans ce numéro du Journal. Ces auteurs citent aussi la possibilité de réactions allergiques ou anaphylactiques comme première raison d'éviter les curares. Il est à noter que le travail de Bouvet et coll. s'est déroulé en France, où l'on se préoccupe beaucoup d'anaphylaxie pendant l'anesthésie. Ceux qui sont en faveur d'une utilisation quasi systématique d'un curare pour l'intubation trachéale proposent que l'obtention de conditions idéales pour l'intubation n'est pas simplement une question d'élégance, mais aussi de sécurité du patient. Des conditions d'intubation médiocres peuvent être accompagnées de toux, de mouvement, de ventilation difficile, de désaturation et d'un risque accru d'inhalation pulmonaire. ${ }^{4}$ Une étude récente a établi une association entre des conditions médiocres et des symptômes de maux de gorge et de raucité de la voix, avec mise en évidence de lésions laryngées. ${ }^{5}$ Avec les données de Bouvet et coll. ${ }^{3}$ et les résultats des études précédentes, devrions-nous nous préoccuper des risques de la curarisation ou de lésions aux cordes vocales?

\section{Conditions d'intubation}

Jusqu'au milieu des années 1990, les chercheurs qui voulaient comparer deux types d'induction sur les conditions d'intubation devaient établir leur propre grille d'évaluation ou en adopter une publiée auparavant. En 1994, un groupe d'experts s'est réuni au Danemark pour proposer une normalisation de plusieurs aspects des études sur les curares, dont les conditions d'intubation. Les recommandations du groupe ont été publiées en $1996,{ }^{1}$ et depuis, la grille d'évaluation des conditions d'intubation a été retenue par presque tous les chercheurs, incluant Bouvet et coll. ${ }^{3}$ En 2005, ces recommandations ont été mises à jour lors d'une réunion tenue à Stockholm et publiées l'an dernier. ${ }^{2}$ Malheureusement, les nouvelles recommandations sur les conditions d'intubations ne sont pas aussi claires et précises que les anciennes, de sorte qu'il est préférable, dans l'avenir, de s'en tenir au texte de 1996.

La grille proposée en 1996 pour les conditions d'intubation est basée sur l'évaluation, dans une étude à double insu, de cinq composantes: la facilité de la laryngoscopie, la position des cordes vocales, le mouvement des cordes vocales, le mouvement des extrémités et la toux. Chaque composante est notée excellente, bonne ou médiocre, selon des critères spécifiques. Par exemple, pour la composante « mouvement des extrémités », on accorde une note "excellente » s'il n'y a aucun mouvement, «bonne » si le mouvement est « discret» et «médiocre » si le mouvement est «vigoureux ». Comme il est facile de constater s'il y a mouvement ou non, la note excellente se démarque aisément des autres. Toutefois, ce qui départage un mouvement « discret » de "vigoureux » pourrait être sujet à interprétation, de sorte qu'il est difficile de séparer le «bon » du "médiocre ». La note finale est la pire des cinq composantes : pour qu'elle soit médiocre, il suffit qu'une seule composante soit jugée médiocre ; elle est bonne si au moins une composante est évaluée comme bonne et aucune n'est médiocre ; et elle est excellente si toutes les composantes sont considérées excellentes.

Dans bien des études, comme celle de Bouvet et coll., ${ }^{3}$ on regroupe les conditions excellentes et bonnes 
sous le vocable « acceptable » et les conditions médiocres sont considérées "inacceptables». Ainsi, la ligne séparant les conditions acceptables de celles qui sont inacceptables devient assez floue et dépend de l'évaluateur. Il peut y avoir cohérence à l'intérieur d'une même étude, mais il est difficile de comparer des études différentes ou d'appliquer les résultats en pratique clinique. Par exemple, Bouvet et coll. rapportent des conditions acceptables chez $95 \%$ des sujets qui n'ont pas reçu de curare. Il est possible qu'un autre évaluateur aurait noté quelques cas de «bonnes» conditions comme étant «médiocres », étant donné le flou entre ces deux catégories. Dans le groupe cisatracurium, on note de bonnes conditions que dans $17 \%$ des cas, le reste étant jugé excellent. Il existe donc moins de situations où un autre observateur aurait pu accorder une note " médiocre ", puisqu'il est peu probable qu'un évaluateur donne une note "excellente » tandis qu'un autre juge ces mêmes conditions comme "médiocres".

On retrouve moins de divergences entre les observateurs lorsque les conditions d'intubation sont excellentes, parce que celles-ci surviennent uniquement lorsqu'il y a absence totale de mouvement. Ainsi, il existe une certaine cohérence entre les études. Par exemple, la plupart des articles rapportent qu'après 1 $\mathrm{mg} \cdot \mathrm{kg}^{-1}$ de succinylcholine, on retrouve d'excellentes conditions dans $70-80 \%$ des cas. $^{6}$

Bien sûr, " excellent » est mieux que seulement «bon " pour nos patients. Un autre avantage à faire la distinction entre «excellent » et «bon » est de pouvoir planifier des études avec des mesures fines et un nombre restreint de sujets. Par exemple, dans l'étude de Bouvet et coll., il n'y avait pas de différence significative entre les groupes quant à la proportion de conditions acceptables (100\% et $95 \%$ avec et sans curarisation, respectivement). En effet, si la différence réelle était de $5 \%$, il faudrait plus de 800 patients par groupe pour obtenir une différence statistiquement significative. Toutefois, en tenant compte des trois notes possibles pour les conditions d'intubation, une analyse des résultats permet de conclure que les conditions d'intubations sont meilleures dans le groupe cisatracurium $(P<0,001)$, et ce, avec 65 patients par groupe. Est-ce pertinent du point de vue clinique ? Selon toute vraisemblance, oui. Si d'excellentes conditions surviennent plus souvent, il y a fort à parier que les conditions médiocres seront plus rares. Et si des conditions médiocres sont présentes dans $5 \%$ des cas, elles surviendront 50 fois pour celui qui réalise 1000 intubations chaque année.

\section{Lésions aux cordes vocales}

L'intubation trachéale comporte un risque très faible de lésions majeures au larynx ou à la trachée, mais ces complications surviennent, en particulier lorsqu'il y a intubation difficile. ${ }^{7}$ Il faudrait une étude avec un nombre énorme de patients pour établir si la curarisation amène plus ou moins de ces lésions. Toutefois, l'intubation trachéale produit des symptômes mineurs, mais fréquents, comme des maux de gorge et une raucité de la voix, et il est probable qu'une intervention qui produit plus de ces effets secondaires mineurs donnerait aussi plus de séquelles plus graves, mais rares, tout comme une proportion élevée de seulement « bonnes » conditions d'intubation est révélatrice d'une incidence plus grande de conditions "médiocres ». C'est la démarche qu'ont entrepris Mencke et coll. ${ }^{5}$ lorsqu'ils ont comparé l'incidence de maux de gorge et de raucité de la voix suite à l'intubation trachéale avec ou sans curare. De plus, un otorhinolaryngologiste expérimenté a évalué les lésions laryngées par un examen fibroscopique. Les conditions d'intubation étaient évidemment meilleures avec curare (atracurium dans ce cas-ci) que sans curare, avec des conditions excellentes dans $42 \%$ et $5 \%$ des cas, respectivement. L'incidence de raucité était plus grande dans le groupe sans curare et était corrélée à la présence de conditions d'intubation médiocres. On retrouvait des lésions aux cordes vocales chez $40 \%$ des sujets qui ont connu des conditions d'intubation médiocres, mais chez seulement 20 $\%$ de ceux dont les conditions étaient excellentes. Les auteurs de cette étude concluaient que «la qualité des conditions d'intubation peut modifier l'incidence de morbidité laryngée ${ }^{5}$

La même équipe de recherche a mené deux autres études sur les lésions laryngées, mais dans lesquelles tous les patients ont reçu un curare. ${ }^{8,9}$ On n'a pas trouvé de différence statistiquement significative quant à l'incidence de maux de gorge, de raucité de la voix ou de lésions aux cordes vocales entre les groupes, probablement parce que l'incidence de conditions d'intubation médiocres n'était pas bien différente entre les groupes et parce que d'autres facteurs, comme la toux à l'extubation, pourrait aussi contribuer à l'apparition d'effets secondaires au larynx. Dans l'étude de Bouvet et coll., l'incidence de lésions laryngées était très faible dans les deux groupes, mais on a examiné seulement les patients dont les symptômes persistaient. L'incidence de raucité de la voix était de 20 à $30 \%$, ce qui est comparable à ce que Mencke et coll. ${ }^{5}$ ont trouvé dans leur première étude chez les patients dont les conditions d'intubation étaient excellentes ou bonnes.

\section{Anaphylaxie}

Sachant que l'administration d'un curare améliore les conditions d'intubation et pourrait diminuer la surve- 
nue d'effets secondaires au niveau laryngé, pourquoi préconiser l'intubation sans curare et pourquoi s'intéresser à des méthodes qui évitent la curarisation ? Lorsque la durée de la chirurgie est brève, une petite dose de curare peut être utilisée, avec de meilleures conditions d'intubation que rien du tout. ${ }^{10}$ On peut diminuer le risque de curarisation résiduelle par l'antagonisation pharmacologique des curares et par un monitorage adéquat. ${ }^{11}$ Bouvet et coll. ${ }^{3}$ retiennent le risque de réaction anaphylactique, qui est perçu comme un problème majeur dans certains pays, comme la France et la Norvège.

L'obsession française concernant les réactions anaphylactiques apparait étrange vue de l'Amérique du Nord ou d'autres pays européens, tout comme le peu de souci qu'on se fait en Amérique du Nord pour le risque allergique peut sembler irréaliste du point de vue français. En France, au cours des 20 dernières années, on a développé un réseau très complet pour le diagnostic des cas. ${ }^{12,13} \mathrm{Il}$ est toutefois difficile de déterminer l'incidence de réactions anaphylactiques à l'aide de telles enquêtes parce que la déclaration des cas pourrait être incomplète, que le dénominateur est incertain et que la fiabilité des tests est inconnue. Néanmoins, l'incidence globale a été estimée dans une fourchette allant de $1: 3000$ et $1: 20000$ anesthésies, avec une incidence plus élevée, peut-être jusqu'à $1: 1500$ pour des substances soupçonnées d'être "allergènes ", comme la succinylcholine et le rocuronium. ${ }^{12-14}$

On s'est aussi intéressé aux réactions allergiques en Australie, au Royaume-Uni et en Scandinavie. En Norvège, le rocuronium est pratiquement disparu des chariots d'anesthésie après que l'on ait relevé 29 réactions anaphylactiques sur un total estimé de 150000 doses en 1997-2000 (1:5 172). ${ }^{15}$ La succinylcholine a été également mise en cause dans un bon nombre de réactions. Pendant la même période, on a recensé seulement sept cas dans les pays voisins (Suède, Finlande et Danemark), pour environ 800000 patients ayant reçu du rocuronium ( $1: 114000)$. D'après les données fournies par un centre danois de consultation pour les allergies, la substance la plus fréquemment impliquée dans les réactions anaphylactiques était la chlorhexidine et non les curares, contrairement à ce qui prévaut en France. ${ }^{16}$ Aux Etats-Unis, les déclarations de réactions allergiques à la Food and Drug Administration sont très rares (environ l 1 1 000000 administrations pour le rocuronium). ${ }^{17}$

Alors que certains ont expliqué ces grandes différences d'incidence aux déclarations incomplètes dans certains pays ou à des variations statistiques, ${ }^{16}$ d'autres ont émis l'hypothèse que certains facteurs dans l'environnement pourraient jouer un rôle. Une étude a été mise sur pied pour rechercher des produits disponibles en Norvège, mais pas dans les autres pays scandinaves, qui pourraient sensibiliser les patients aux curares. ${ }^{18}$ On a retrouvé des anticorps IgE à la succinylcholine chez $0,4 \%$ des donneurs de sang en Norvège, mais chez aucun en Suède. Après avoir passé plusieurs produits au crible, les auteurs de l'étude se sont tournés vers la pholcodine, un médicament contre la toux retrouvé dans les sirops en vente libre en Norvège, mais pas en Suède. Une sensibilité croisée avec les curares pourrait survenir. Fait intéressant, la pholcodine est disponible en France, en Australie et au Royaume-Uni, là où il y a beaucoup d'intérêt pour les réactions allergiques, mais pas au Canada et aux Etats-Unis. ${ }^{18}$

Peut-on attribuer à la pholcodine ce qui semble être des différences dans l'incidence de réactions anaphylactiques entre les pays ? Jusqu'à présent, les preuves sont circonstancielles, mais la piste est intéressante. D'autres facteurs pourraient être impliqués et le nombre de déclarations pourrait être gonflé. De toutes façons, il est intéressant de penser que quelque chose d'aussi saugrenu que la présence ou l'absence d'un sirop contre la toux en vente libre dans une collectivité pourrait influencer notre façon de considérer l'intubation trachéale et la pratique de l'anesthésie.

\section{Conclusion}

La raucité de la voix et les lésions laryngées sont fréquentes et ces complications ont été reliées à des conditions d'intubation médiocres. Ces effets secondaires mineurs sont sans doute des indicateurs de complications moins fréquentes, mais plus graves, de l'intubation trachéale. De la même façon, un type d'induction de l'anesthésie qui produit des conditions d'intubation qualifiées de bonnes, sans être excellentes, est plus susceptible de donner des conditions médiocres chez certains patients qu'une induction qui donne d'excellentes conditions chez la plupart des sujets. Ainsi, lorsqu'on vise des conditions excellentes, pas seulement bonnes, on réduit la possibilité de lésions laryngées et le meilleur moyen d'y parvenir est de curariser. La raison la plus impérieuse d'éviter la curarisation est le risque perçu de réactions allergiques et il existe des preuves assez faibles que ce risque pourrait dépendre du pays où l'on pratique. Il est dommage qu'une forme plus bénigne, mais plus fréquente, de réaction allergique n'ait pas été décrite, car elle pourrait servir d'indicateur d'événements plus graves, comme c'est le cas pour des lésions laryngées. Les anesthésiologistes qui utilisent les curares pour intuber devraient continuer à le faire : si des réactions allergiques surviennent à une fréquence de $>1: 5000$, de tels événements reviendraient au moins chaque année dans un hôpital 
de taille moyenne et chaque anesthésiologiste serait au courant du problème. Ceux qui choisissent de procéder à l'intubation sans curare à cause de la possibilité de réactions anaphylactiques devraient développer les habiletés et l'expérience pour viser des conditions d'intubation non pas seulement bonnes, mais excellentes.

\section{References}

1 Viby-Mogensen J, Engbaek J, Eriksson LI, et al. Good clinical research practice (GCRP) in pharmacodynamic studies of neuromuscular blocking agents. Acta Anaesthesiol Scand 1996; 40: 59-74.

2 Fuchs-Buder T, Claudius C, Skovgaard LT, Eriksson LI, Mirakbur RK, Viby-Mogensen J; 8 $^{\text {th }}$ International Neuromuscular Meeting. Good clinical research practice in pharmacodynamic studies of neuromuscular blocking agents II: the Stockholm revision. Acta Anaesthesiol Scand 2007; 51: 789-808.

3 Bouvet L, Stoian A, Jacquot-Laperriere S, Allaouchiche $B$, Chassard D, Boselli E. Laryngeal injuries and intubating conditions with or without muscular relaxation: an equivalence study. Can J Anesth 2008; 55: 674-84.

4 Warner MA, Warner ME, Weber JG. Clinical significance of pulmonary aspiration during the postoperative period. Anesthesiology 1993; 78: 56-62.

5 Mencke T, Echternach M, Kleinschmidt S, et al. Laryngeal morbidity and quality of tracheal intubation: a randomized controlled trial. Anesthesiology 2003; 98: 1049-56.

6 Donati $F$. The right dose of succinylcholine. Anesthesiology 2003; 99: 1037-8.

7 Domino KB, Posner KL, Caplan RA, Cheney FW. Airway injury during anesthesia: a closed claim analysis. Anesthesiology 1999; 91: 1703-11.

8 Mencke T, Echternach M, Plinkert PK, et al. Does the timing of tracheal intubation based on neuromuscular monitoring decrease laryngeal injury? A randomized, prospective, controlled trial. Anesth Analg 2006; 102: 306-12.

9 Mencke T, Knoll H, Schreiber JU, et al. Rocuronium is not associated with more vocal cord injuries than succinylcholine after rapid-sequence induction: a randomized, prospective, controlled trial. Anesth Analg 2006; 102: 943-9.

10 Barclay K, Eggers K, Asai T. Low-dose rocuronium improves conditions for tracheal intubation after induction of anaesthesia with propofol and alfentanil. Br J Anaesth 1997; 78: 92-4.

11 Naguib M, Kopman AF, Ensor JE. Neuromuscular monitoring and postoperative residual curarization: a meta-analysis. Br J Anaesth 2007; 98: 302-16.
12 Laxenaire MC, Mertes PM; Groupe d'Études de Réactions Anaphylactoïdes Peranesthésiques. Anaphylaxis during anaesthesia. Results of a two-year survey in France. Br J Anaesth 2001; 87: 549-58.

13 Mertes PM, Laxenaire MC, Alla F; Groupe d'Études de Réactions Anaphylactoïdes Peranesthésiques. Anaphylactic and anaphylactoid reactions occurring during anesthesia in France in 1999-2000. Anesthesiology 2003; 99: 536-45.

14 Malinovsky JM, Decagny S, Wessel F, Guilloux L, Mertes PM. Systematic follow-up increases incidence of anaphylaxis during adverse reactions in anesthetized patients. Acta Anaesthesiol Scand 2008; 52: 175-81.

15 Laake JH, Rottingen JA. Rocuronium and anaphylaxis - a statistical challenge. Acta Anaesthesiol Scand 2001; 45: 1196-203.

16 Garvey LH, Roed-Petersen J, Menne T, Husum B. Danish Anaesthesia Allergy Centre - preliminary results. Acta Anaesthesiol Scand 2001; 45: 1204-9.

17 Bhananker SM, O'Donnell JT, Salemi JR, Bishop MJ. The risk of anaphylactic reactions to rocuronium in the United States is comparable to that of vecuronium: an analysis of Food and Drug Administration reporting of adverse events. Anesth Analg 2005; 101: 819-22.

18 Florvaag E, Johansson SG, Oman H, et al. Prevalence of $\mathrm{IgE}$ antibodies to morphine. Relation to the high and low incidences of NMBA analphylaxis in Norway and Sweden, respectively. Acta Anaesthesiol Scand 2005; 437-44. 